\title{
Hacia una definición de Sedentarismo
}

\author{
Dr. Tomás Romero \\ “... y dejáme muriendo \\ un no se qué \\ que quedan balbuciendo..."
}

San Juan de la Cruz. Prólogo, Cántico Espiritual

Recibido el 9 de octubre de 2009, aceptado el 20 de noviembre de 2009

Rev Chil Cardiol 2009; 28: 409-413

\section{Introducción}

Desde el punto de vista antropológico, el término "sedentarismo" (del Latin "sedere", o la acción de tomar asiento) se ha utilizado para describir la transición de una sociedad nómade a otra establecida en torno a un lugar o región determinada. Este cambio se ha relacionado a la evolución de grupos sociales en que el sustento dependía de la caza y recolección de frutos, a una organización social-productiva basada en la agricultura y en la domesticación de animales ${ }^{1}, 2$. Es probable que en las formas de vida nómades se requería un mayor gasto energético en las actividades diarias para satisfacer las necesidades individuales y del grupo social, en contraste a la sociedad sedentaria, aunque en ésta el gasto energético de los individuos en los estratos sociales más bajos era sin duda mayor que el de los de niveles más altos, lo que probablemente se acentuó aun más en las etapas iniciales de la revolución industrial. Más adelante, el advenimiento de los métodos mecanizados de transporte y trabajo, y el progresivo desarrollo de la sociedad industrial avanzada han reforzado las características de la sociedad sedentaria en todos los estratos sociales reduciendo cada vez más las oportunidades de gasto energético en la vida diaria.

Al mismo tiempo, el proceso de industrialización de la cadena alimenticia ha generado distorsiones importantes alrededor del globo: de la caza de aves silvestres se ha evolucionado al pollo McNugget ${ }^{1}$. En la sociedad sedentaria no sólo se han reducido las oportunidades del gasto energético a través del ejercicio físico, sino que al mismo tiempo ha aumentado el consumo excesivo de calorías baratas, con el consiguiente problema creciente de la obesidad a nivel mundial ${ }^{3}$. El concepto de sedentarismo desde el punto de vista médico debe por lo tanto extenderse a este doble significado, y enfocarse al desajuste calórico que hoy día afecta a gran parte de la humanidad. Aunque el nomadismo persiste en algunas regiones aisladas (grupos en Mongolia, Mauritania, Etiopia, Sudan, Kenya, Mali, Matto Grosso e incluso Escandinavia), la mayoría ha ido adoptando una forma mixta de organización y conducta social, y posiblemente evolucionarán hacia estructuras social-productivas predominantemente sedentarias ${ }^{2}$.

\section{Actividad Física y protección de la salud.}

El concepto de que la actividad física es un indicador de vida saludable ha existido desde los comienzos de las civilizaciones más antiguas. Referencias aisladas al respecto datan desde hace más de 3.000 mil años (Timoteo, libro del Éxodo, Antiguo Testamento), y abundan a partir de las civilizaciones Griega y Romana 4 . Sin embargo, sólo en los últimos 60 años se han acumulado evidencias científicas del efecto protector del ejercicio tanto en individuos aparentemente sanos como en aquellos

Correspondencia: Dr. Tomás Romero

Dirección 831 Adella Ave, Coronado, California 92118

Correo Electrónico: tomas.romero@sharp.com 
portadores de enfermedad cardiovascular 5, 6, 7, 8, 9, 10, $11,12,13,14,15$. Una cantidad creciente de información ha provisto una base plausible para entender los posibles mecanismos envueltos en ese efecto protector del ejercicio físico: movilización de células progenitoras del endotelio y apoyo a la integridad vascular, inhibición de factores pro-inflamatorios, aumento de la sensibilidad a la insulina, supraregulación de enzimas anti-oxidantes, factores neurovegetativos $16,17,18,19$.

Un concepto clave relacionado con el efecto protector del ejercicio es la cantidad de energía consumida al efectuarlo. La forma de expresarla ha sido mediante el consumo de oxígeno $\left(\mathrm{O}_{2}\right)$ de la masa corporal en la unidad de tiempo (equivalente a medir el trabajo de un motor a combustión por la cantidad de gasolina consumida en un período determinado). Este consumo ha sido expresado en unidades Met (1 Met es la cantidad de $\mathrm{O} 2$ consumida por kilogramo de peso corporal en un minuto por un individuo en reposo), y equivale a $3.5 \mathrm{ml} \mathrm{O} 2 / \mathrm{kg} / \mathrm{min}$. Se ha determinado que 1 Met corresponde aproximadamente a $1 \mathrm{kcal} / \mathrm{kg} /$ hora producidas en reposo, que a su vez equivale a $4.184 \mathrm{kj} / \mathrm{kg} / \mathrm{hora}$, unidad que ocasionalmente ha sido utilizada en este tipo de mediciones 20, 21.

Los trabajos pioneros de Morris y Paffenbarger 5, 6 , analizando el primero la actividad física efectuada por los cobradores y choferes en los buses Londinenses, y el segundo, la de los estibadores y oficinistas en el puerto de San Francisco sugirieron una base cuantitativa del efecto protector del ejercicio. Paffenbarger y colaboradores ${ }^{6}$ estimaron que el riesgo relativo $(\mathrm{RR})$ de muerte por infarto agudo del miocardio en los trabajadores portuarios que gastaban menos de $8.500 \mathrm{kcal} / \mathrm{semana}$ era de $1.80(p<0.01)$, en un período de observación de 22 años. Posteriormente, evaluando a través de cuestionarios la energía gastada por ex -alumnos de las Universidades de Pennsylvania y Harvard en actividades de tiempo libre (las kcal semanales empleadas caminando, o practicando algún deporte) en un período de 6-10 años, determinaron que aquellos con un gasto menor de $2.000 \mathrm{kcal}$ por semana tenían un $64 \%$ de mayor riesgo de un evento coronario 7,8 .
A partir de estos trabajos, que relacionaban cuantitativamente el ejercicio físico con su efecto protector, numerosas publicaciones han confirmado hallazgos similares.

La mayoría de esta información ha sido obtenida a través de cuestionarios detallando la actividad realizada en las últimas 24 horas y extrapolando esta información al resto de la semana. Uno de los más utilizados ha sido el International Physical Activity Questionnaire (IPAQ) que ha sido traducido a numerosos idiomas incluyendo el Castellano. En éste, se estima la actividad física en cuanto a su duración, frecuencia e intensidad, definiéndose de esta manera, niveles bajo, moderado y alto, que corresponden a 3.3 Mets (marcha normal), 4.4 Mets (marcha mas rápida) y 8.0 Mets (marcha vigorosa o trote), respectivamente.

Este instrumento puede utilizarse para estimar el gasto energético de todas las actividades diarias, conociéndose su duración, frecuencia e intensidad, y el peso del individuo, en base a los valores promedios previamente establecidos para cada una de ellas 20, 21, 22, 23.

\section{Actividad Física Beneficiosa, ¿qué es lo que realmente cuenta?}

Algunos autores han definido como "sedentarios" a quienes gastan en actividades de tiempo libre menos del $10 \%$ de la energía total empleada en la actividad física diaria. Un informe del US Department of Health and Human Services publicado en 1995 recomendando agregar un gasto adicional de alrededor $150 \mathrm{kcal}$ al consumo energético diario para lograr mayores beneficios de salud probablemente dio sustento a esa definición, ya que estas $150 \mathrm{kcal}$ adicionales (cifra cercana al $10 \%$ del gasto calórico diario) pueden consumirse caminando alrededor de 30 minutos, 3 o más días de la semana. Hoy en día esta recomendación ha sido extendida a un ejercicio equivalente a 30 minutos de caminata todos los días de la semana24, 25, 10.

Basados en esa definición de sedentarismo, varios estudios hechos en diferentes regiones han señalado cifras de prevalencia de alrededor de $84 \%$ en los hombres y $89 \%$ en las mujeres 24,25 . En nuestro medio la Encuesta Nacional de Salud, publicada el año 2003 consideró como activa "a la 
persona que practica una actividad física fuera del horario de trabajo equivalente a 30 minutos 3 veces por semana", y "sedentarios" a quienes no cumplían esta meta ( $88 \%$ de los hombres y $91 \%$ de las mujeres); evidentemente la energía gastada en los otros dominios de actividad no fue considerada en esta encuesta26.

¿Cómo lograr un gasto calórico extra semanal que cumpla los objetivos de protección cardiovascular? Para muchos en los que el uso de tiempo libre diario para desarrollar actividades de ejercicio físico resulta difícil o imposible, la oportunidad de hacerlo los fines de semana aparece como una opción válida si se logra una meta de $1.000 \mathrm{kcal}$ o más, de acuerdo a información derivada del estudio de los ex-alumnos de Harvard.

De acuerdo a los hallazgos de este estudio, quienes lograban esa meta tenían, en un período de 5 años de seguimiento, un riesgo significativamente menor de mortalidad por toda causa $(R R=0.41, p<0.01)$ que aquellos con baja actividad física semanal $(<500$ Kcal/semana) ${ }^{27}$.

Sin embargo es la cantidad total de energía consumida en la actividad física diaria junto a la intensidad de ésta lo que muestra mayor relación con los beneficios de salud observados. Estos han sido confirmados en múltiples estudios hechos en diferentes regiones y grupos étnicos $6,7,8,9,10,11,12$, 13, 27, 28, 29).

Un estudio reciente hecho en Noruega (comunicado en el Congreso de la Sociedad de Cardiología Europea 2009, Barcelona, España) mostró la influencia beneficiosa de la combinación de cuantía e intensidad del ejercicio aeróbico en hipertensos, no solo en el control de la presión arterial, sino que además del perfil lipídico (aumento del colesterol HDL) y de la función endotelial (aumento de la respuesta vasodilatadora) 29 .

En nuestro medio, en un estudio presentado en el Congreso Chileno de Cardiología de 2008, en el que se evaluó el consumo energético en todos los dominios de actividad diaria en hipertensos controlados en consultorios periféricos de la región Metropolitana utilizando el cuestionario IPAQ, se encontró que niveles bajos de actividad ocurrían en sólo el $5.1 \%$ de los hombres y el $23.7 \%$ de las mujeres 30 . En contraste con la Encuesta Nacional de Salud del año 2003, el 58.4\% de los hombres alcanzaron un nivel alto de actividad física, logrando especialmente en el dominio correspondiente a la actividad laboral y de transporte, a pesar de que sólo el $7.6 \%$ de la energía gastada ocurrió en actividades de tiempo libre. Estos individuos hubieran sido clasificados como "sedentarios", de acuerdo a la definición señalada anteriormente. En la programación de campañas de salud públicas destinadas a reducir las conductas sedentarias, aparece entonces como un instrumento indispensable el estudio de la distribución del gasto energético en todos los dominios de actividad de la población a considerar.

Una publicación reciente publicado, en que se evaluó la actividad física mediante el cuestionario IPAQ demostró que los individuos con un nivel más alto de actividad física y gasto energético presentaban una mayor cantidad de células progenitoras endoteliales circulantes y una respuesta vasodilatadora mayor que en aquellos con un nivel de actividad física baja, sin una clara relación con el vigor del ejercicio realizado (el ejercicio vigoroso fue responsable de $<$ del $10 \%$ del total de energía gastada en todos los grupos) 28 .

Este estudio, al igual que los previamente señalados, enfatizan la importancia de considerar el gasto total de energía en la evaluación de la influencia de la actividad física en la salud.

\section{Conclusiones}

Las recomendaciones actuales, provenientes de las fuentes más autorizadas sobre la materia, de aumentar el tiempo libre dedicado al ejercicio físico (equivalentes a 30 minutos de caminata diaria) 11, 22 se encuentran a menudo limitadas por la tendencia casi universal (tanto en las sociedades mas desarrolladas como aquellas en diferentes grados de transición socioeconómica) de utilizar cada vez más el tiempo libre en actividades sedentarias (tiempo dedicado a la TV, Internet, transporte mecanizado). La importancia del gasto energético global en las actividades diarias suele ser minimizada por una interpretación errónea de esas recomendaciones, reflejada por la tendencia a considerar como 


\section{T. Romero}

"sedentarios" a quienes no satisfacen una cierta cuota de ejercicio físico en tiempo libre, a pesar de que muchos en realidad pueden alcanzar un consumo energético alto a través de las actividades laborales, domésticas o de transporte. Además de las campañas destinadas a convencer a la población a emplear una mayor parte de su tiempo libre al ejercicio físico, es importante que se incentiven las oportunidades existentes en la vida diaria para aumentar el gasto energético a través del ejercicio: utilizar las escaleras en vez del ascensor, caminar o usar la bicicleta como medio de transporte, bajarse del Metro o del bus una estación o un paradero antes, estacionar el auto a varias cuadras del trabajo, etc. y emplear parte del tiempo libre de los fines de semana a actividades de ejercicio físico. Pero todo este esfuerzo no tiene mucho sentido si no va acompañado de programas y campañas en las escuelas, centros laborales públicos y privados, y a través de los medios de difusión para reducir la ingesta calórica excesiva. En definitiva, además de intentar establecer patrones o definiciones más o menos aproximadas de gasto energético para decidir quienes son o no "sedentarios" (en último término, desde el punto de vista histórico y socio-cultural, lo son hoy en día casi todos los habitantes del globo), resultará más efectivo dedicar recursos y esfuerzos en el desarrollo de programas y campañas para que la mayoría de la población logre metas protectoras de alimentación y ejercicio físico de acuerdo con la realidad y las evidencias disponibles. Es la existencia de un profundo desequilibrio entre ingesta y gasto calórico quizás el rasgo que mejor define al sedentarismo desde el punto de vista de la salud.

\section{Referencias}

1. POLLAN M. "The Omnivore's Dilemma". New York, The Penguin Press, 2006

2. ELWOOD W."Nomads at the Crossroads", New Internationalist, No.266, April 1995.

3. WORLD HEALTH ORGANIZATION 2008 REPORT. Contacts: PUSKA P, NISHIDA C, PORTER D. Global Strategy on Diet, Physical Activity and Health. Obesity and Overweight. Available from: http://www.who.int/ dietphysicalactivity/media/en/gsfs_obesity.pdf.

4. HAGGARD HW. The Doctor in History. New York, NY, Dorset Press, 1989.

5. MORRIS JN, HEADY JA, RAFFLE PA, ROBERTS CG, PARKS JW. Coronary heart disease and physical activity at work. Lancet 1953; 2: 1053-57.

6. PAFFENBARGER RS, LAUGHLIN ME, GIMA AS, BLACK RA. Work activity of longshoremen as related to death from coronary heart disease and stroke. New Eng J Med 1970; 282: 1109-1114.

7. PAFFENBARGER RS JR, WING AL, HYDE RT. Chronic disease in former college students. XVI. Physical activity as an index of heart attack risk in college alumni. Am J Epidemiol 1978; 108:161-175.

8. PAFFENBARGER RS JR, HYDE RT, WING AL, LEE IM, JUNG DL, KAMPERT JB. The association of changes in physical-activity level and other lifestyle characteristics with mortality among men. N Engl J Med. 1993; 328: 538-545.

9. WANNAMETHEE S, SHAPER A, WALKER M. Changes in physical activity, mortality, and incidence of coronary heart disease in older men. Lancet 1998; 351: 1603-8.

10. PHYSICAL ACTIVITY GUIDELINES ADVISORY
COMMITTEE. Physical activity guidelines advisory committee report Washington, DC: US Department of Health and Human Services, 2008.

www.health.gov/paguidelines/Report/Default.aspx.

11. ROSENGREN A, WILHELMSEN L. Physical activity protects against coronary death and deaths from all causes in middle-aged men. Evidence from year follow-up of the primary prevention study in Göteborg. Ann Epidemiol. 1997; 7: 69-75.

12. HASKEL W, LEE IM, PATE RR, POWELL K, BLAIR SN, FRANKLIN BA, et al. Physical activity and public health, update recommendations for adults from the American College of Sport Medicine and the American Heart Association. Med Sci Sports Excerc 2007, 39:1423-34.

13. HU G, JOUSILAHTI P, ANTIKAINEN R, TUOMILEHTO J. Occupational, commuting, and leisure time physical activity in relation to cardiovascular mortality among Finnish subjects with hypertension. Am J Hypertens 2007, 20:1242-50.

14. O'CONNOR GT, BURING JE, YUSUF S, GOLDHABER SZ, OLMSTEAD EM, PAFFENBARGER RS, et al. An overview of randomized trials of rehabilitation with exercise after myocardial infarction. Circulation 1989; 80: 234-244.

15. ROMERO T. Cardiac Rehabilitation as a first step in the secondary prevention of coronary artery disease. Rev Med Chile 2000; 128: 923-934.

16. KASAPIS C, THOMPSON PD. The effects of physical activity on serum $\mathrm{C}$-reactive protein and onflammatory markers. A systematic review. JACC 2005; 45:1563-9.

17. MCLAUGHLIN T, ABBASI F, LAMENDOLA C, LIANG L, 
REAVEN G, SCHAAF P, et al. Differentiation between obesity and insulin resistance in the association with C-reactive protein. Circulation 2002; 106: 2908-12.

18. POWERS SK, JII LL, LEEUWENBURGH C. Exercise training-induced alterations in skeletal muscle antioxidant capacity: a brief review. Med Sci Sports Exerc 1999; 31: 987-97.

19. WERNER N, KOSIOL S, SCHIEGL T, AHLERS P, WALENTA K, LINK A, et al Circulating Endothelial Progenitor Cells and Cardiovascular Outcomes N Engl J Med 2005; 353: 999-1007.

20. AINSWORTH BE, HASKELL WL, WHITT MC, IRWIN M L, SWARTZ AM, STRATH SJ, et al. Compendium of Physical Activities: an update of activity codes and MET intensities. Medicine \& Science in Sports \& Exercise 2000; 32: S498-S516.

21. AINSWORTH BE: The Compendium of physical activities tracking guide. http://prevention.sph.sc.edu/tools/docs/ documents_compendium.pdf.

22. CRAIG CL, MARSHALL AL, SJÖSTRÖM M, BAUMAN AE, BOOTH ML, AINSWORTH BE, et al. International Physical Activity Questionnaire: 12 country reliability and validity Med Sci Sports Exerc 2003; 35:1381-1395

23. PATE RR, PRATT M, BLAIR SN, HASKELL WL, MACERA CA, BOUCHARD $C$, et al. Physical activity and public health. A recommendation from the Centers for Disease Control and Prevention and the American College of Sports Medicine. JAMA 1995; 273:402-7.

24. BERNSTEIN MS, MORABIA A, SLOUTSKIS D. Definition and prevalence of sedentarism in an urban population. Am J Public Health. 1999; 89: 862-867.

25. GAL DL, SANTOS AC, BARROS $H$. Leisure-time versus full-day energy expenditure: a cross-sectional study of sedentarism in a Portuguese urban population. BMC Public Health 2005, 5:16.

26. MINISTERIO DE SALUD, GOBIERNO DE CHILE. Primera Encuesta Nacional de Salud. Informe Final, Ministerio de Salud (MINSAL), Santiago, Chile 2004 http://www.minsal.cl/ici/destacados/Folleto\%20FINAL.pdf

27. LEE IM, SESSO HD, OGUMA Y, PAFFENBARGER RS JR. The "weekend warrior" and risk of mortality. Am J Epidemiol 2004; 160: 636-41.

28. LUK TH, DAI YL, SIU CW, YIU KH, CHAN HT, FONG DY, et al. Habitual physical activity is associated with endothelial function and endothelial progenitor cells in patients with stable coronary artery disease. Eur J Cardiovasc Prev Rehabil 2009; 16: 464-471.

29. MOLMEN HANSEN HE, STOLEN T, TJONNA AE, AAMOT IL, SCHJERVE EKEBERG I, TYLDUM G. Aerobic interval training decreases blood pressure more than moderate intensity training in patients with essential hypertension.

http://127.0.0.1:9080/ESC09/view.y?nu=ESC09L1_2009P3802

30. BRAVO M, KOCH E, SANDOVAL D, ROMERO T. Niveles de actividad fisica en pacientes hipertensos: un estudio exploratorio en atención primaria. Rev. Chil. Cardiol. 2008; 3: 309. 